\title{
Initial Experiments using Game-based Learning Applied in a Classical Knowledge Robotics in In-Person and Distance Learning Classroom
}

Márcio Mendonça ${ }^{*}$, , Rodrigo Henrique Cunha Palácios ${ }^{1,2}$, Ivan Rossato Chrun ${ }^{3}$, Diene Eire de Mello ${ }^{4}$, Henrique Cavalieri Agonilha ${ }^{5}$, Elpiniki Papageorgiou ${ }^{6}, K^{\prime}$ onstantinos Papageorgiou ${ }^{6}$

${ }^{1}$ Programa de Pós-Graduação em Engenharia Mecânica (PPGEM-CP), Universidade Tecnológica Federal do Paraná, Av. Alberto Carazzai, 1640, Cornélio Procópio, Brazil

${ }^{2}$ Programa de Pós-Graduação em Informática (PPGI-CP), Universidade Tecnológica Federal do Paraná, Av. Alberto Carazzai 1640, Cornélio Procópio, Brazil

${ }^{3}$ Departamento de Engenharia Química, Universidade Estadual de Maringá, Av. Colombo 5790, Maringá, Brazil

${ }^{4}$ Programa de Pós-Graduação em Educação (PPEDU- UEL), Universidade Estadual de Londrina, Av. Colombo 5790, Maringá, Brazil

${ }^{5}$ Departamento de Acadêmico de Computação (DACOM), Universidade Tecnológica Federal do Paraná, Av. Alberto Carazzai 1640, Cornélio Procópio, Brazil

${ }^{7}$ Department of Energy Systems, University of Thessaly, Geopolis Campus, 41500 Larissa, Greece

A R T I C L E IN F O

Article history:

Received: 16 March, 2021

Accepted: 13 July, 2021

Online: 27 July, 2021

Keywords:

Digital games

Game-based learning

Mobile robotics

Robotic manipulators

Autonomous robotics

\begin{abstract}
A B S T R A C T
This paper addresses experiments with Scratch-developed games in the robotics introduction course at the Federal University of Technology-Paraná. It aims at assisting learning of classical and initial robotics concepts. This proposal, similar to the classic $80 \mathrm{~s}$ war tanks game on Atari 2600, was developed using an autonomous vehicle. In the first experiment, applied to the class 2019/2, the students (players) had to battle against another autonomous tank developed (in two different ways, in Person and Distance Learning), using keyboard inputs to control their tank. In this game, the students were asked to create states machine models while were being introduced to fundamental concepts such as pose, other basic notions concerning controlled and autonomous robots, and the hierarchy of actions. At the end of the games, a questionnaire answered by the students extracted valuable findings of the examined concepts. In 2021/1 class, the second and third experiments were applied. The former was an extension of the first experiment, using autonomous parking cars. The latter was inspired by the classic Pong game, with the addition of more degrees of freedom (DOF). In this case, the player attempts to reach and catch a ball through the operation of a robotic arm with two rotating joints, using keyboard inputs. Each block or scenario will become more complex, and the student has time to perform a task. In the case of the third experiment, the concepts including 2-D workspace, multiple solutions, inverse, and direct kinematics were explored. Delivery rates for the first and second experiments were $90 \%$ and $80 \%$, respectively. Even though three individual experiments were investigated, the single objective was achieved: the implementation of modern didactic tools to deliver critical pedagogical concepts to students in the robotics class.
\end{abstract}

\section{Introduction}

The first 20 years of 2000 have been highlighted by the development and creation of computer technologies. This study is

${ }^{*}$ Corresponding Author Márcio Mendonça, Email: mmendoncautfpr@gmail.com www.astesj.com

https://dx.doi.org/10.25046/aj060425 devoted to the integration of certain aspects of these technologies to either the working or leisure areas of everyday human life. In the field of leisure, games have been transformed into digital, triggering increased attention to users of all ages around the world, 
while the extended popularization of the web helped in this direction. Electronic games are characterized by an enormous variety of levels in terms of their type and difficulty, targeting a wider age group, no longer being a niche market [1], as it used to be in the past.

This paper is an extended version of the previous work published in ILSA 2020 [2] approaching game-based learning. The proposal of active methodologies such as game-based learning aims to involve students by establishing a process that implies action-reflection-action and not just internalization of what has been exposed (as is the case in in-person classes) [3].

With Game-based learning, students learn while playing. Thus, making the learning process more enjoyable, causing a positive effect on cognitive development. Games are combined with traditional classes because the conventional learning process can be monotonous, and game-based learning can improve students' motivation to learn. It is not just about using games to review and reinforce concepts [4].

The games include many problem-solving features, adding elements of competition and opportunity. That is, the student player needs to deal with an unknown result, choose between several paths to an objective, construct a context of the problem, and collaborate with several players [4].

Among the benefits of games implementation in learning, according to [5], the following attributes should be mentioned:

- Games can easily attract the attention of individuals across various demographic boundaries (for example, age, sex, ethnicity and educational status).

- Games can assist young people in setting their goals as they can provide feedback and reinforcement or record changes in human behaviour.

- Games offer fun and excitement to the players. Hence, it is not difficult to attract and maintain a person's attention.

- Games also offer the chance to participants to explore their curiosity and new challenges, thus stimulating their motivation for learning.

On that basis, gamification is the practice of using elements of game design, mechanics and thinking in non-game activities to motivate participants. Gamification in the field of education exploits, among others, sets of games rules, players' experiences, and cultural roles in shaping students' behaviour [4]. Thus, it uses points, badges, ratings, and incentives to engage students in the learning process.

The benefits of gamification in education are [4]:

- Improved learning experience.

- Enhanced learning environment.

- Instant Feedback.

- Promotion of behavioral changes.

- Feasibility to integrate into different learning needs.
Nowadays, the utilization of novel learning technologies has overcome the conventional problems of distance, time, and cost in learning. However, the lack of student motivation is a problem that e-learning still faces. The application of gamification in e-learning is being used aiming at giving participation and increasing student motivation. Within the same learning content, the characteristics of different users and static gamification elements do not increase the expected motivation. To overcome this problem, gamification must be adapted to the characteristics and needs of the learners [6].

The development of computers, tablets, and smartphones with enhanced capabilities derived from improved processing power and low cost has boosted the popularity and wide use of digital games among young people. On this basis, researchers have recently focused on exploring the intrusion of games into the teaching and learning process. In this context, the authors, through the examined scenario, investigate how technological means could improve the teaching-learning process using digital games.

This approach is based on traditional teaching and learning methods, whereas it exploits diverse aspects of the learning process [7]. Additionally, it utilizes question games, where students are rewarded with marks when delivering the correct answers, thus creating active cooperation and healthy competition, which stimulates the learning process; this process is also known as gamification. This gamification scenario has been appropriately applied to exhibit the contribution of games in the learning processes in students' interests [8].

The successful integration of games in education to complement traditional learning is highly attributed to the valuable features of games, including the playful aspects, interactivity, feedback, problem-solving, experimentation, competition, and students' engagement in learning [7, 9]. To instantiate the methodology, the work $[10,11]$ presents, in addition to learning the proposed concepts, that the games favour students' cognitive and social development through the solution of problems and cooperation between them. An example that can be cited in the literature is the work with children playing with robots. Some elements are relevant in game development and were used in this article, shown as follows.

As a motivation for this research at university level, we can mention the work that uses game-based learning in conjunction with other methods as a teaching technique for children. In [12], a novel teaching structure assisted by a computer was presented for teaching maths in the 5th grade. Based on an award-winning curriculum program, this approach uses music and body gestures to develop certain connections between mathematical concepts and culturally inspired metaphors. Utilizing Virtual Augmented Reality (VAR) and sensors, the presented approach deals with the successful transformation of the class from traditional to digital.

Another factor that supports this research is the fact that the Brazilian Computer Society (SBC) considers the basic concepts of computing as important as those of mathematics, philosophy, physics, and other sciences for contemporary life. Thus, computer science, robotics and digital games have found new adherents with meaningful pedagogical experiences.

It is not the scope of this research to carry out any statistical analysis, only to present roughly the percentage of students who 
suggested having abstracted the classical knowledge of robotics in each of the proposed experiments.

Digital games, since their introduction in 1974, have taken tremendous and rapid steps towards improving players' overall gaming experience and involvement. Actually, game developers intended to keep people engaged in playing their game uninterruptedly, targeting new goals every time, being determined to experience new challenges. Even though today's children have a different view towards the use of video and computer games than games were meant to offer, students still show an optimistic attitude characterized as interested, competitive, cooperative, results oriented. At the same time, they actively seek information, fundamentals (which is the purpose of this research, toward robotics) and even solutions [1].

As for the application of games based on Atari, a relevant consideration suggests a possible motivation of students in the application of games, even if it is worth mentioning that: the use of DeepMind Technologies Limited acquired by Google in 2014, has been breaking all records of Atari games and is capable of challenging any human to a match [13]. This motivated students about the importance of digital games in intelligent computational systems and for learning robotics. That said, the students answered questionnaires and developed state machines.

This article is divided into five sections. Section 1 presents the introduction and a brief review of the literature. Section 2 presents the theoretical aspects of games and learning. Section 3 presents the development of the research developed. The results are presented in Section 4, and, finally, in Section 5, the overall conclusions and discussions are presented.

\section{Games and learning: theoretical aspects}

This approach aims at improving learning, using the motivating effects of the elements and techniques of digital games. Student engagement is the criterion for integrating gamification into the learning process and therefore serves as an essential measure for its effectiveness. However, gamification should not be restricted in considering only games' main concepts such as points and leaderboards, which significantly reduce its educational value and the desired impact on students, who constitute the main target of this attempt [14].

Among the advantages of game-based learning that motivates the current study is the ability to provide learners with the understanding of concepts in a practical way, taking the student to a higher level of involvement with his learning in a more dynamic way. In this context, the work of [15] presents a model under study which illustrates the fact that an intense engagement in learning has a strong effect on human body as dopamine and serotonin do. Specifically, this neural model was based on the assumptions that dopaminergic activity increases as the expected reward increases and serotonergic activity increases as the expected cost of an action increases.

Following a brief history review regarding learning and technology, the view of Robert McClintock, Frank Moretti and Luyen Chou is devoted to the evolution of technology which goes side by side with the change and transformations in teaching and learning. Originally, education and training were a process of imitation and training - "picking up a stone and playing with the animal." If you are unable to do this the first time, practice several times until you succeed. "No, do it this way." The practice has become a way of playing to make this repetitive skill-based learning bearable and memorable. This type of "demonstration and practice" learning requires good coaches, usually in an individual relationship. This is how people learn to play sports, play musical instruments, and master other physical skills. In the most basic, not even language is necessary, e.g., athletes and musicians are often skillfully trained by people who do not or barely speak the same language [1].

According to [16], since the 1980s, some researchers carried out certain studies concerning the use of games in education and its benefits and approaches. In [17], the authors reported the popularization of gamification started only in 2010 . The term gamification refers to the use of game elements, as aesthetics, game thinking and mechanics, in non-game-related contexts to involve people, motivate action, improve learning, and solve problems.

It also involves several concepts, such as rewarding and punishing the players. It has several connotations, such as the case of this research in which games are developed to assist in the learning process, in commercial games, or even in the commercial area. For example, in [6], the authors use a Systematic Literature Review (SLR) to explore adaptive gamification in terms of frameworks and methods proposed, as well as other research components. The first step is to define the research question (RQ) and then to search the literature published in popular scientific journal databases. Twenty-five selected articles were finally reviewed, in which the authors identified three elements that comprise the proposed framework. These are adaptive gamification engine, adaptive component, and gamification display. Additionally, eleven types of methods were implemented in adaptive gamification. Among them, Felder-Silverman Learning Style Model (FSLSM) is the most popular method. As for the components of adaptive gamification, four were mined, namely: player/learner profiles, learning style, behavior, and skill/knowledge.

Specifically, the following examples are related to this research. The authors in [18] conclude that in recent years, interest in the theme has been increased to such extend so that a theoretical game model was developed for educational purposes. In this case, not any particular game element could be used for gamification, except a subtly combination of elements that are used to contextualize learning. The initiative behind the game development was the integration of various types of activities into this game, as well as assisting teachers in working with modernized learning content.

Another study considers games as powerful experiences that exploit motivation and engagement [17]. In particular, the deployment of simplified elements reduces project complexity concerning badges, levels, points, and leaderboards, that fail to involve students and damage any existing interest for the learning process. A thorough consideration regarding game design must be given in gamification, apart from just implementing game components successfully. However, gamification is a broader 
concept used in different areas of knowledge, for example, the management area [6].

In [19], it was observed that educators could increase the feedback mechanisms by exploiting specific game design elements by applying continuous feedback, visual cues, frequent question and answer activities as well a progress bar. Students are also prompted to interact with the content, experience real-life case studies, make decisions for specific tasks, and have realistic consequences for making wrong or unsatisfactory choices. In this way, they are getting involved within the game flow, which attracts students' attention. In conclusion, satisfactory learning outcomes come when facts are embedded adequately inside a game-based story rather than in a passive text format.

In the example of Sheldon's work [20], the professor of a higher education institution gamified his electronic games development class. Students' marks were starting from zero and increasing with their results. Moreover, there was an increase in the number of game-based activities against the traditional assessments. These activities had the form of missions to defeat enemies and were assigned to groups of students. The outcome of each mission was assessed and formed the student's final grade. Throughout this learning process, it was observed that the average grades of the students were notably improved at the end.

Gee also noted that students perform better when a level system is used in the game-based learning process. The players are more inspired when they apply what they have learned and get the corresponding feedback after completing each level. Each subsequent level requires, after all, skills acquired at previous levels [21]. The authors in [22] conclude that the game's dynamics are improved when levels or progress bars are involved. In addition, storytelling is another game design aspect that highly contributes to the successful integration of games into the learning process. Some examples of these types of games include SimCity, where players follow the story of building a city from scratch, as well as Monopoly, where the story behind the game is to become rich buying and selling property in every round, trying to avoid the risk of losing everything [16].

After an in-depth review of the available literature, certain characteristics were found mainly in [16,17,19-21] regarding the successful development of games integrated into learning environments. These include Freedom to Fail, Quick Feedback, Progression and Counting stories.

\section{Development}

There are several approaches that deal with the integration of digital games in learning environments. The simplest paradigm refers to the utilization of simple games used exclusively in the classroom whereas, more complex examples focus on the design of digital game concepts that are put into practice but are not necessarily used as learning simulators in virtual or real educational environments [7].

It is not the scope of this work, but it is possible to use board games, for example, as a learning method. Several research works can be found in the literature which study board games-based learning. More specifically, the authors in [7] propose cooperative learning as a means to design an educational course based on the board game. According to the curriculum, pre-service teachers cooperate in developing and delivering a board game prototype.

Because of the game's simplicity, the tutorial with the game instructions was presented during the beginning of the class in Brazilian and Portuguese (Brazil is the native language) and is presented in the metadata of this work.

In the first stage, a group discussion in an online platform took place where the participants were working on the fulfillment of specific activities according to the curriculum. Next, a questionnaire evaluated the performance of the pre-service teachers in the particular task. Based on the results produced, all participants' groups were able to successfully design games in line with the academic disciplines that are played sufficiently and without difficulties. Additionally, teachers' self-efficacy was improved through the process of cooperative learning.

Another study in this area that contributes to learning [23] presents a novel structure devoted to the general board game (GBG). GBG configures boardgame's standard interfaces, states and AI agents. This structure offers cooperation with various agents in different games and defines the parts of board game coding. GBG is particularly suitable for arbitrary $1,2,3$ or multiplayer board games.

This work proposes three different games developed individually. The first one is devoted to designing a tanking battle game based on a classic Atari 2600. This game includes the definition of some concepts regarding robotics discipline fundamentals. These are the pose concept that complements the Cartesian coordinates $(\mathrm{x}, \mathrm{y})$ and the angle, which denotes the object's orientation. Two more games are also presented: one consisting of a manipulator arm and a ball, while the last one deals with car parking.

Goals: each experiment has its own distinct goals. In tanks, the objective is to destroy the opponent; in the robotic arm, the goal is to reach the ball and, finally, in the valet, a parking space must be found.

Rules: In the case of tank war, the opponent must be destroyed before the player is destroyed; in other games, the objectives must be accomplished in the shortest time possible.

The proposed digital game was built on the Scratch platform, which was developed by the Massachusetts Institute of Technology (MIT) team in 2007. Scratch is among the most accessible programming languages as it uses a graphical interface and does not require programming skills on any other programming language [24]. The scope of this game is to insert blocks, as shown in the example in Figure 1.

The methodology of the learning process was initially the presentation of various games, such as Pong, which was the first game of the era. Then an explanation followed on how the games work, and next, the commands and objectives were exhibited. In the next phase, the students were introduced to relevant areas of robotics and/or virtual agents related to the tank war game and continued with activities concerning the following two experiments presented. 


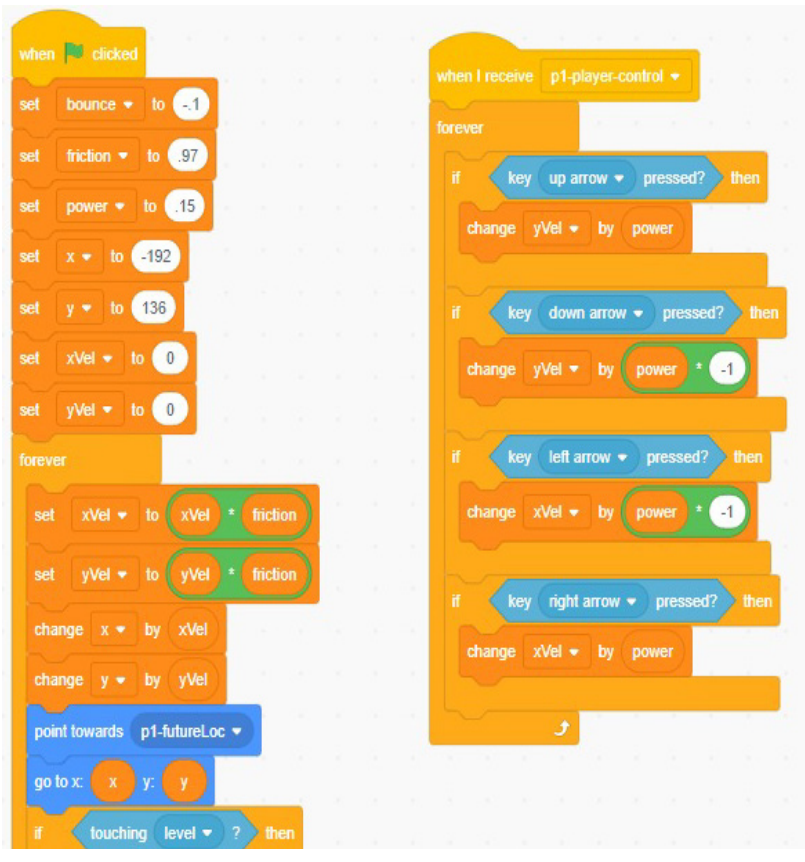

Figure 1: Screen example using Scratch.

\section{Results}

Three games built on Scratch were presented in different classes. The tanks war (experiment 1) and the autonomous valet (experiment 2) games were presented to the 2019/2 class, and various responses were collected. In sequence, the game with two robotic arms (experiment 3 ) was illustrated, in which students tried to simply catch a ball (target) by rotating the two arms joints (2 degrees of freedom, GDL) with the help of a keyboard. Concerning the first experiment, 27 students were involved. However, just a small number of results for all three experiments is presented for the purposes of this study due to the limited workspace.

\subsection{Experiment 1}

The purpose of this exercise was to assist students in understanding certain robotics concepts that concern the need for autonomy and hierarchy when actions are taken. Priority is given to some control actions in the scenario, such as obstacles avoidance and targeting the opposing tank. Additionally, new routines can be applied in the development of future works based on intelligent computer systems. For example, the opponent can become an autonomous entity by implementing intelligent computational methods based on Fuzzy logic. Fuzzy Cognitive Maps can help in this direction in the future, as it provides low computational complexity $[25,26]$.

In other words, this experiment added the abstraction of a robotic architecture inspired by fundamental concepts of Brooks' subsumption architecture [27]. The notion of priority when a robot, or in this game's case a virtual robot (bot), must prioritize its actions, such as staying alive, avoiding enemy attacks and obstacles before shooting the enemy.

In the initial phase of this game, as shown in Figure 2, both tanks are guided by players. The main objective of the first tank is to destroy the second by shooting at it while trying to avoid other fixed and mobile obstacles (opponent's shots). The next phase of the game design includes determining the game rules and then establishing the possible states of the tanks.

The game rules are defined according to possible damage suffered by the tanks. The game has a winning condition for the first player when he manages three hits to the opponent's tank. One point of damage is attributed either by a shot or physical contact with an obstacle. The distinct states of a tank are the following:

- Moving freely.

- Shooting.

- Avoiding a fixed obstacle.

- Avoiding a moving obstacle (opponent's shot).

According to the exercise proposal in the experiment developed in the seventh period of Control Engineering and Automation, the following tasks need to be accomplished by students:

- Develop tank's finite state machine.

- Identify the tank's position (coordinates) and pose (angle with the $\mathrm{x}$-axis).

In this experiment, we had an abstraction of the mobile robotics concepts of approximately $80 \%$ for one class and $90 \%$ for another, despite having initial results with applications in only two classes. Objectivized concepts like hierarchy and pose in mobile robotics were abstracted, suggesting that this game is promising for introducing the basic concepts of programmed and mainly autonomous mobile robotics.

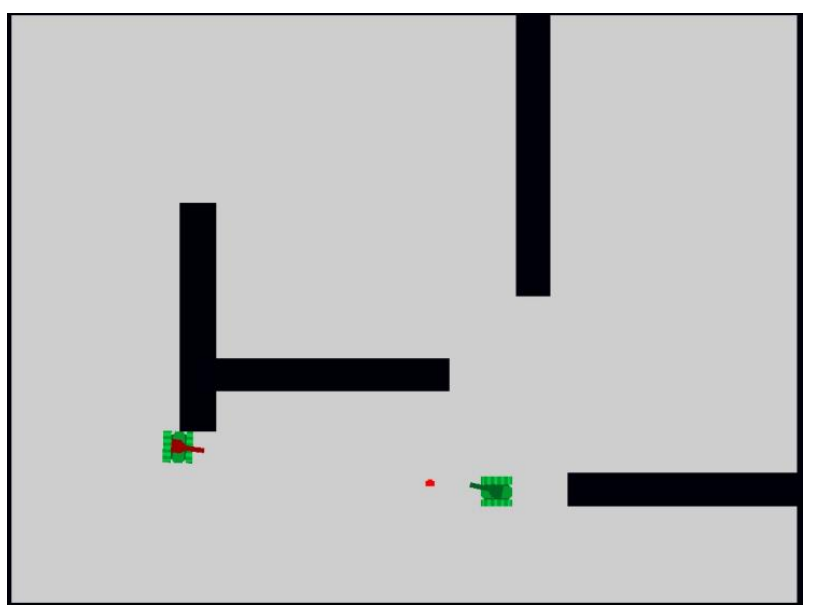

Figure 2: Representation of the first game

Based on the proposal, each student was asked to give a figure of the state machine and a description of the events in a doc or pdf file. In addition to the graphical representation, students were also asked to answer a group of questions to identify whether the nature of the activity made it possible for them to perceive aspects and concepts proposed. Such an activity can be considered as a Subjective Modeling when capturing the impression of the student in the short-term memory as listed below:

a) Is it possible to distinguish between the programmed and the autonomous control? Explain. 
b) Is it possible to perceive the robot's hierarchy during the game, and in what way?

c) Is an exemplified angle during the battle necessary, in addition to the $\mathrm{x}, \mathrm{y}$ coordinates?

d) Is It possible to perceive the different states, attacks and defences?

e) Develop a state machine that models the player's actions to defeat the opponent.

One exemplary and one complete answer provided by the students are listed below for each question:

Question a:

- Student 1: Yes, because the user-controlled tank responds to the given commands, whereas the autonomous only responds to the events following its logical construction.

- Student 2: It is possible. The opponent's tank moves without any human intervention. On the other hand, the player's tank is controlled at each cycle by the keyboard.

Question b:

- Student 1: The hierarchy is noticed as the robot tank is looking for its own defense, and then is trying to destroy the opponent with the intention to win. Our tank has no hierarchy since it is controlled by the user.

- Student 2: Hierarchy is perceived when activating opponents, avoiding obstacles and the opponent's attack for keeping its integrity (game's objective).

Question c:

- Student 1: The angle is used to increase the control precision. For example, position $\mathrm{x}=0, \mathrm{y}=0$ is where the tank is located in the environment. When $X=1$, the tank will not necessarily move forward, but it may be rotated.

- Student 2: The question was not answered.

Question d: only two students answered the questions. However, the state machine abstraction was the main objective of this experiment.

Student 1: Student 1 provided a satisfactory interpretation of the robot's movement finite-state machine, shown in Figure 3. The possible states are: 1 - shoot avoidance; 2 - stopped; 3 - chasing the enemy; 4 - aiming at the enemy tank; 5 - shooting the enemy and 6 - obstacle avoidance.

Only two students were inserted due to a large amount of space for a more accurate analysis. However, students were chosen at random, and their responses can provide feedback on their learning from the most complex experiment, the tank battle. Other students have already managed to develop state machines for game strategy, which are shown below.

The response by the student is presented below.

a) Do not press anything.

b) Press arrow keys. c) Mouse left-click.

d) Spin the mouse.

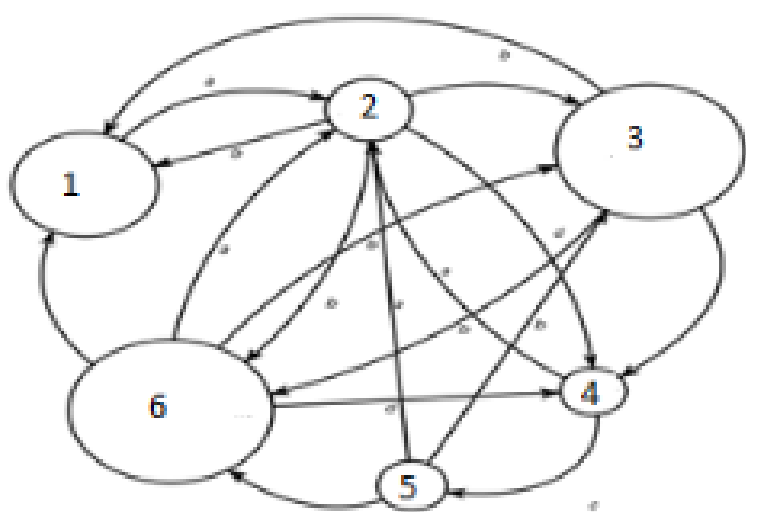

Figure 3: Finite-state machine by Student 1.

On the other hand, it is worth noticing that student 2 (Figure 4) provided a more complete interpretation than those submitted by the rest of the class. It seems to be more accurate and closer to the actual operation of the tanks in the game.

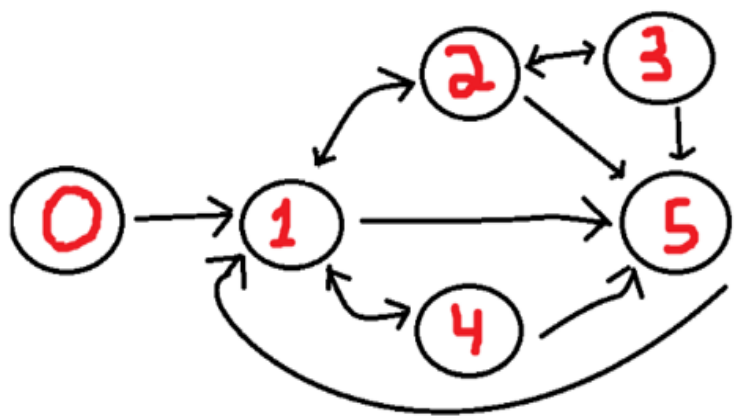

Figure 4: Finite-state machine by Student 2

- State 0: The game is stopped and has not yet started.

- State 1: The game started; tank not moving.

- State 2: The tank is moving.

- State 3: The tank moves, aiming and firing.

- State 4: The tank is stopped, aiming and firing.

- State 5: The tank is dead, awaiting re-entering to the game.

- State $0 \rightarrow 1$ : Press the button to start the game.

- State $1 \rightarrow 2$ : Press the directional buttons to move the tank.

- State $2 \rightarrow 1$ : Release the movement buttons. Tank in motion.

- State $2 \rightarrow 3$ : Press the shoot button to target. Tank in motion.

- $\quad$ State $3 \rightarrow 2$ : Release the fire button. Tank in motion.

- State $1 \rightarrow 4$ : Press the fire button while aiming at the target. Tank is stopped.

- States 1, 2, 3, 4 $\rightarrow$ 5: The tank got damage.

- State $5 \rightarrow 1$ : The tank respawns after an $\mathrm{x}$ amount of time has passed. 
Regarding student 3 , he developed the state machine but left the vocabulary unfinished. Through this process, it emerges that the students have delivered various interpretations. Additionally, the students seemed to comprehend specific concepts in the field of robotics and their autonomous mode as well.

The graphical representation that student 4 provided (see Figure 5) illustrates the finite-state machine of the game combined with the respective vocabulary, offering a satisfactory interpretation of the activity concept. Although different interpretations emerged from students, these interpretations were similar concerning the game strategy. Overall, the number of different ways for learning assessment in each of the experiments needs to be highlighted.

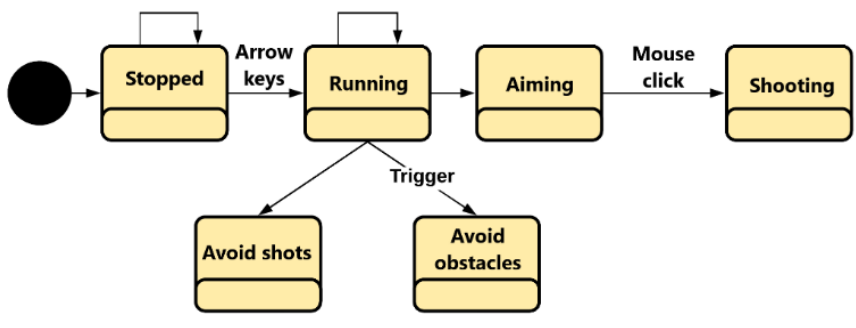

Figure 5: Finite-state machine by Student 4

In this tank warfare experiment, using the state machine was necessary due to different behaviours in combat, such as an attack, defence, avoiding obstacles, enemy shots, and chasing the enemy, among others. However, in the last experiment, four questions were enough to assess students' learning. In general, each experiment has a complexity level, whereas different evaluation methods were used.

State machines have been promising in terms of students' abstraction. Most of the students, about $40 \%$, did not even know the state machines yet (because they are students of an Automation and Control course, whose robotics discipline is offered from the seventh period on). So, the results of this experiment showed promising abstraction of the game's strategy, especially the concepts of robotics involved, mentioned in the text.

New experiments took place in 2021 with distance learning due to the pandemic (University policy to mitigate Covid contagion). The difficulty of experimenting compared to previous ones before the pandemic was much greater [24]. It was necessary to do a live meeting and provide videos of the game's action to help students abstract the strategy and do their required tasks. Figure 6 shows details of the video provided to students.

UTFPR-CP, a federal university (in Portuguese Universidade Tecnológica Federal do Paraná Campus Cornélio Procópio), gave a subsidy to low-income students to purchase computers. In addition, it also promoted aid in cash to low-income students, which was also maintained during the pandemic. Only three examples (see Figures 7, 8, 9) will be presented, which took place in a distance learning class during the pandemic.

The ICT Panel COVID-19 (2020), which took place with young people aged 16 or over, showed that the cell phone was mentioned by $22 \%$ of users in social classes A and B, $43 \%$ of users in class $\mathrm{C}$ and $54 \%$ of users in classes $\mathrm{D}$ and $\mathrm{E}$. The inequalities of student access to connected devices are striking. The same document also points out: three-quarters of Internet users aged 16 or over in classes $\mathrm{D}$ and $\mathrm{E}$ (74\%) used the network exclusively by cell phone, a percentage that was $11 \%$ among users of classes A and B (no reference because it was written in Portuguese).

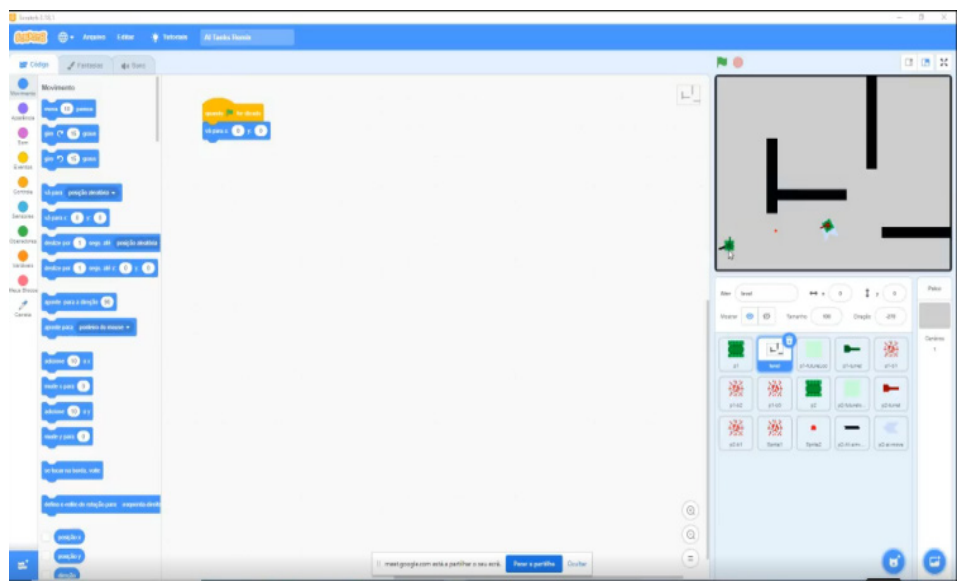

Figure 6: shows details of the video provided to students.

Student 1 (Distance Learning):

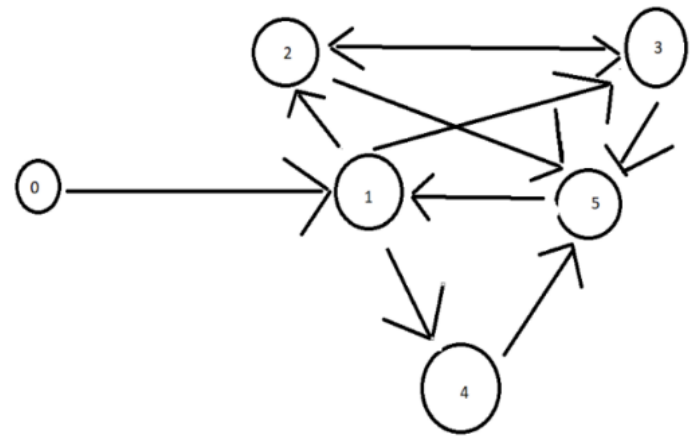

Figure 7: Finite-state machine by Student 1 (distance learning)

Vocabulary state machine.

- $\quad$ State $0 \rightarrow 1$ : Start the game.

- $\quad$ State $1 \rightarrow 2$ : The player moves the tank without shooting.

- State $1 \rightarrow 3$ : The player moves the tank and shoots.

- State $1 \rightarrow 4$ : The player stops the tank and shoots.

- $\quad$ State $4 \rightarrow 5$ : The tank was stopped and died.

- $\quad$ State $2 \rightarrow 5$ : the tank was moving and died.

- $\quad$ State $3 \rightarrow 5$ : the tank was moving and shooting and died.

- State $5 \rightarrow 1$ : The tank was dead and reappeared.

- State $3 \rightarrow 2$ : Tank moving stopped shooting.

- $\quad$ State $2 \rightarrow 3$ : Tank moving started shooting.

Student 2 (Distance Learning):

Vocabulary state machine.

- State $0 \rightarrow 1$ : Starts the game, with the tank stopped. 
- $\quad$ State $1 \rightarrow 2$ : Tank is moving.

- $\quad$ State $2 \rightarrow 3$ : Tank is moving and firing.

- State $3 \rightarrow 5:$ The tank, while firing, dies and awaits reappearance.

- $\quad$ State $5 \rightarrow$ 1: The tank was dead and reappeared.

- $\quad$ State $1 \rightarrow 4$ : Stopped Tank aims and shoots.

- $\quad$ State $4 \rightarrow 5$ : Tank was stopped and died.

- $\quad$ State $5 \rightarrow$ 1: The tank was dead and reappeared.

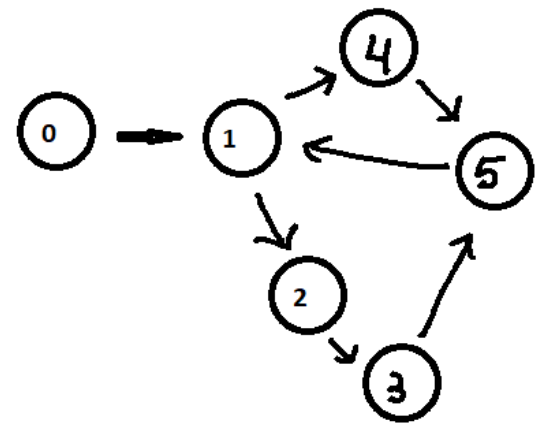

Figure 8: Finite-state machine by Student 2 (distance learning)

Student 3 (Distance Learning):

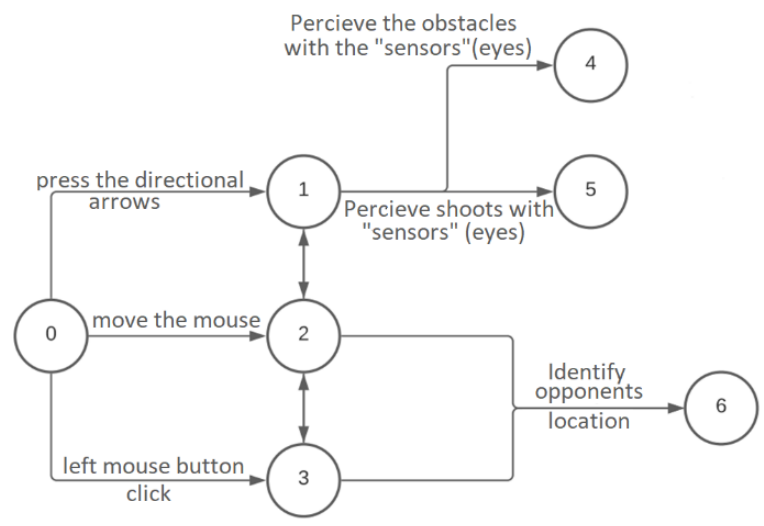

Figure 9: Finite-state machine by Student 3 (distance learning)

Vocabulary state machine.

- State 0: Game start.

- State 1: Player walking.

- State 2: Player aiming.

- State 3: Player shooting.

- State 4: Player dodging obstacles.

- State 5: Player dodging shots.

- State 6: Player hitting opponent.

The first two results were compatible with the experiments carried out in the classroom. The third result presented was one of the best in abstraction, if not the best of all the experiments presented in distance learning and on-site teaching. In addition to the states, the figure shows the events used to control the player's tank.

\subsection{Experiment 2}

Another simpler game was produced on Scratch, contributing to this work. In this game, the student must drive a car to find a parking space (autonomous valet), as shown in Figures 10 and 11. It was held in the same class (2019/2) following the tank war game.

It was also introduced in the robotics discipline, aiming to present students a brief notion of the maneuver difficulty, even if performed on the keyboard, by an autonomous valet using Fuzzy logic as an example.

The coexistence of several agents or virtual robots in the same scenario was emphasized. It is noteworthy that in the discipline of robotics, students have a posterior example of an autonomous valet. This game reinforces the concepts of tank warfare: autonomy and hierarchy of control actions. That is, it is necessary to avoid the cars reaching a parking space.

One student's answer: despite being a relatively simple activity for human beings, the game allowed us to observe the difficulties of developing an autonomous system, which would make these maneuvers without any human intervention. It helped us to understand a little more about autonomous robotics.

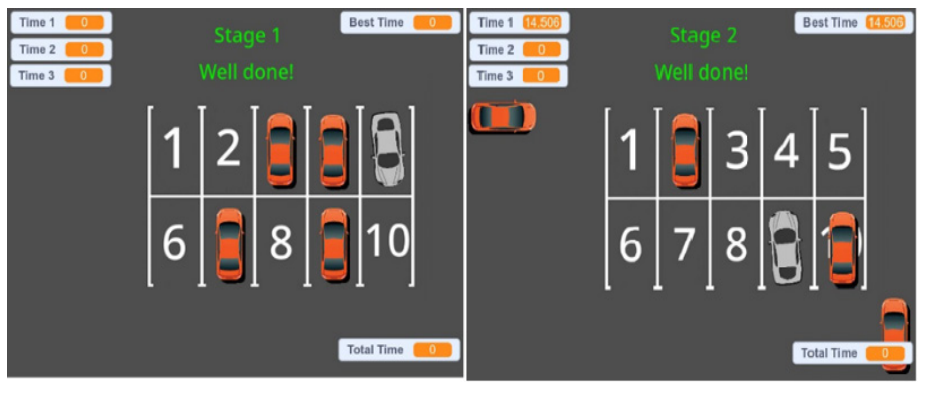

Figure 10: Student 1 answers; initial stages

Compared to Experiment 2, different results were obtained and aided in the autonomous vehicle abstraction. However, two of several examples will be cited in which can be seen that the second student was faster than the first one, as shown in Figures 10 to 13. Nevertheless, practically all students were successful in all three stages. The first objective is to help develop an autonomous valet through Fuzzy logic, for example, fuzzy cognitive maps [28].

The course has already clarified the difference between programmed and autonomous robots, a relevant concept in robotics [29].

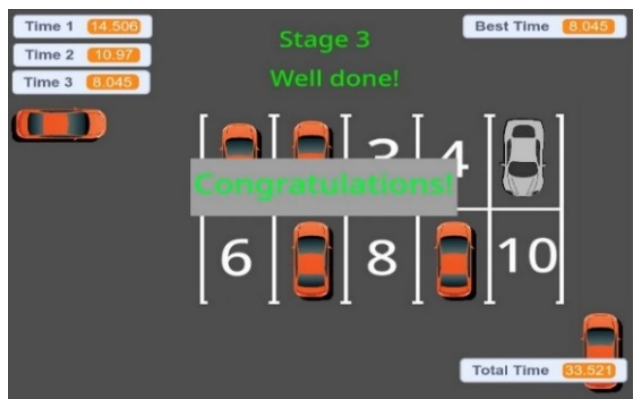

Figure 11: Student 1 answers; final stage 


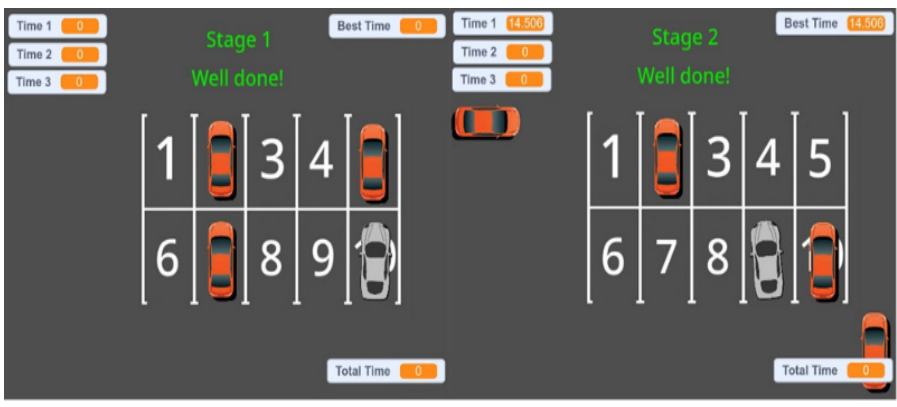

Figure 12: Student 2 answers; initial stages

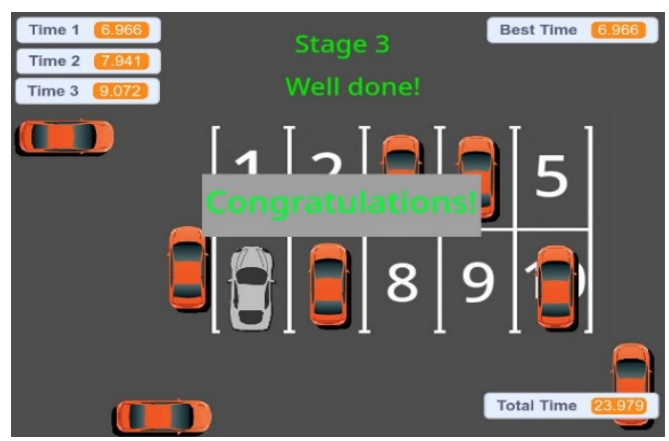

Figure 13: Student 2 answers; final stage

\subsection{Experiment 3}

The robotic arms game (experiment 3 ) was applied in the class of 2020/1, which has approximately 23 students. The game works as follows: a ball (target) appears on the scene at random as shown in Figures 14 and 15. The student's goal is to try and catch it using the cursors.

The game objective is to emphasize on direct and inverse cinematics concepts and the action radius (the robot inserts in a 2D environment). Future work could also implement a 3D game addressing a sphere or a search surface. In addition to the objectives, a classic problem regarding robotics manipulation became clear to students: the multiple solutions that can occur.

According to the figures presented, the game strategy is oriented to reaching three targets in sequence within the space area. However, the ball's movement cannot be achieved in certain situations, especially when the robotic arm is out of reach. It should be mentioned that the robotic arm has two degrees of freedom and also have rotational joints.

Due to workspace restrictions in this paper, the presentation of the answers to the provided questions will be limited, and only a few examples will be presented. It also needs to be highlighted that the results illustrated below were randomly selected to maintain the authenticity and truthfulness of the current research. Experiment 3 involves the following questions:

a) Is it possible to use more movements to catch the ball?

b) Would the movement become more natural if the joints move at the same time?

c) Does the game have direct or inverse kinematics?

d) Are there inaccessible positions of the arm?
In the meantime, the results in Figure 15 should be considered as they are the second successful attempt for student 2 . The answers of five students are shown below for each of the four questions.

Question a:

- Student 1: Since the arm has two DOF, it is possible to have more than one solution.

- Student 2: It is possible for some ball positions, according to the image of the first line, in the same position, that there is more than one way to capture it. However, in the second line, there is only one possibility.

- Student 3: Yes, the movement is more harmonious when the joints move simultaneously, as this situation results in faster movements to reach the ball, more agile and more natural compared to a real human arm.

- Student 4: Yes, with the combination of the angles and the ball's position.

- Student 5: Yes, more than one solution is possible.

Question b:

- Student 1: Yes, this process would require greater computational power, but it would have a more harmonious movement closer to the human arm movement.

- Student 2: It would be, but it is easier to use keys to move both together simultaneously in the game.

- Student 3: Yes, it is possible to have different solutions to catch the ball, as each player has the freedom to choose the arm's movements by joining different angles for each joint but reaching the same point in the Cartesian plane.

- Student 4: Yes, it mimics the human body more.

- Student 5: Yes.

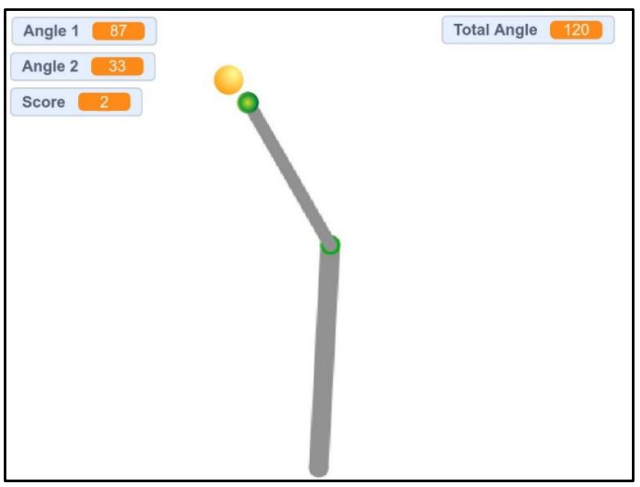

Figure 14: Student 1 result: first attempt

Question c:

- Student 1: Inverse, as the movement orientation of the joints occurs through the ball's position.

- Student 2: Inverse kinematics because the arm has a desired position and orientation (ball). 
- Student 3: The game has inverse kinematics, as the player must define the joints' positions after a given ball orientation.

- Student 4: Inverse, the arm needs to follow the position of the given ball.

- Student 5: Direct, because the angles are already given, so the position of the arm can be found. In the game, we are going after the target "ball" visually.

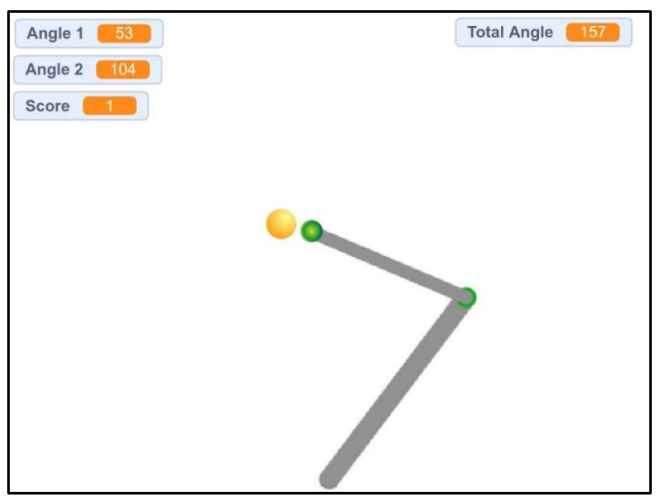

Figure 15: Student 1 result: second attempt

Question d:

- Student 1: Yes.

- Student 2: Yes, according to the figures, the ball travels practically the entire space of the screen and some points that are not unattainable.

- Student 3: The question was not answered.

- Student 4: Yes, so that a key is needed in the game to reset the ball.

- Student 5: Yes, then there is the ball moving.

The given answers emerge that they were similar since students could identify the problem just by playing the game. The correct answer to the fourth question is that the arm cannot reach the ball (target) because of its structure. Moreover, student 4 gave a more consistent answer to the second question, explaining his response assertively. Overall, the game seemed to have its objective fulfilled. In specific, considering a sample corresponding to approximately $20 \%$ of the class, it emerges that some answers were more competent than others. However, it is concluded that there was an abstraction of the contents and objective foundations.

Figures 14 and 15 illustrate in a more precise way the structure of the game's components and how the robotic arm changes its position to reach its target (ball), according to the game's random logic. The experiment was carried out in pairs, and the students had to repeat it three times and search for the target. The game attempted to leverage active learning [26] through objective concepts visualization. Additionally, the game demanded multiple solutions from students, as they had to find the target near or similar positions with different angles.

To provide a further understanding of the game, it is necessary to examine the arm's angles. Based on the representation inspired by the geometric model of MathWorks (see Figure 16), the angle $\theta 1$ is formed by the intersection of Arm 1 (L1) and the X-axis. The angle $\theta 2$ is formed by the continuation of Arm 1 (L1) and Arm 2 (L2), considering that $(\mathrm{X}, \mathrm{Y})$ is the desired position.

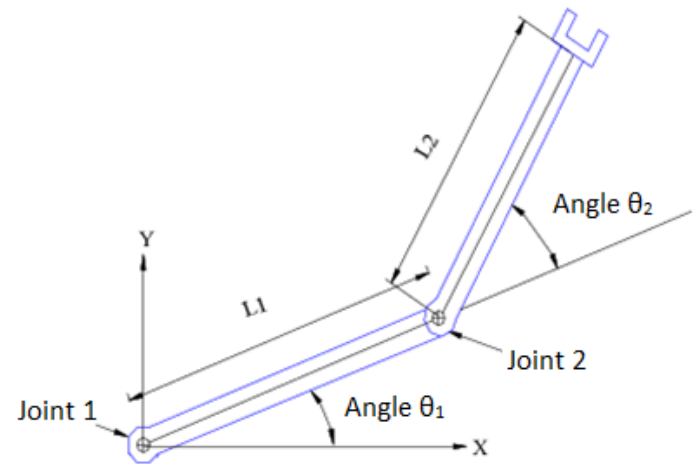

Figure 16: Representation of 2-DOF robotic manipulator.

\section{Conclusion}

The results, although initial, suggest the feasibility of the Scratch's proposals based on classic games of the $80 \mathrm{~s}$. The most important part of the experiments was the fact that the students experienced and became familiar with significant concepts in the domain of robotics through playing games. Among the produced outcomes was students' perception who considered the class "lighter" and liked the experiments. In short, the results were seemed convincing in terms of game-based learning since over $80 \%$ of the students had shown interest in these experiments, even if they were relatively simple. Therefore, the application of this methodology is considered quite promising.

In addition, it was possible to carry out some examples of experiments 1 and 3 in the distance learning modality, with a slight reduction in the percentage of students' interest, approximately $70 \%$. Furthermore, experiment 3 had one of the most complex state machines and one of the best (if not the best) of the tank battle game, as mentioned above, which reinforces the promising results of the method.

The participation and motivation of the students in general in both classes was over $80 \%$. According to the few results of the experiments, it can be observed that the objectives of introducing the basic concepts of robotics initially proposed were abstracted by the students with an even higher percentage than the motivational. That is approximately $85 \%$.

Future works can emphasize the control of the opponent's tank and the appearance of obstacles like trees in the tank battle game. For the robotic arm, a 3D arm can be introduced to increase the difficulty of reaching the target significantly. Thus, the solution of the inverse kinematics would be more complex and the number of solutions to the problem. And finally, future work can be oriented to the development of realistic prototypes that would increase the difficulty of the game and introduce and utilize soft computing based on Fuzzy Cognitive Maps (FCMs). Considering that this intelligent technique has low computational complexity, it could be exploited to construct prototypes of low computational and financial cost, such as Arduino. Finally, as mentioned, the next generation of the games may also deploy other programming platforms, for instance, UNIT. Overall, considering the promising 
results of distance learning, it would be interesting to conduct new experiments.

\section{Conflict of Interest}

The authors declare no conflict of interest.

\section{Acknowledgment}

The authors gratefully acknowledge the Federal University of Technology - Paraná, campus Cornélio Procópio.

\section{References}

[1] M, Prensky, Digital game-based learning. McGraw-Hill, 2001.

[2] M. Mendonça et al., "Digital Game-Based Learning in a Robotics Course," in 202011 th International Conference on Information, Intelligence, Systems and Applications (IISA), 1-7, 2020, doi: 10.1109/IISA50023.2020.9284366.

[3] R. Al-azawi, F. Al-faliti, M. Al-blushi, "Educational gamification vs. game based learning: Comparative study," in International Journal of Innovation, Management and Technology, 7(4), 132-136, 2016, doi: 10.18178/ijimt.2016.7.4.659.

[4] M. Ebner, A. Holzinger, "Successful implementation of user-centered game based learning in higher education: an example from civil engineering," Computers \& Education, 49(3), 873-890, 2007, doi:10.1016/j.compedu.2005.11.026.

[5] F. Rozi, Y. Rosmansyah, B. Dabarsyah, "A Systematic Literature Review on Adaptive Gamification: Components, Methods, and Frameworks," in 2019 International Conference on Electrical Engineering and Informatics (ICEEI), 187-190, 2019, doi:10.1109/ICEEI47359.2019.8988857.

[6] S. Sena, et al., "Aprendizagem Baseada em Jogos Digitais: A Contribuição dos Jogos Epistêmicos na Geração de Novos Conhecimentos," in Revista Novas Tecnologias na Educação (RENOTE), 14(1), 1-11. 2016.

[7] S. Freitas, "Are games effective learning tools? A review of educational games," Educational Technology \& Society. 21(2), 74-84, 2018.

[8] P. F. C. Santana, D. X Fortes, R. A. Porto, "Jogos digitais: a utilização no processo ensino aprendizagem," Revista Científica da FASETE, 1, 218-229, 2016.

[9] T.-H. Tsai, H.-C. Lin, K.-C. Huang, "Digital Game-Based Learning on Digital Archives: A Case Study of Taiwanese Classical Poems," in 2012 IEEE Fourth International Conference on Digital Game and Intelligent Toy Enhanced Learning. 132-134, 2012, doi:10.1109/DIGITEL.2012.36.

[10] N. P. Zea, J. L. G. Sanchez, F. L. Gutierrez, "Collaborative Learning by Means of Video Games: An Entertainment System in the Learning Processes," in 2009 Ninth IEEE International Conference on Advanced Learning Technologies, 215-217, 2009, doi:10.1109/ICALT.2009.95.

[11] A. Barmpoutis, et al., "Exploration of Kinesthetic Gaming for Enhancing Elementary Math Education Using Culturally Responsive Teaching Methodologies," in 2016 IEEE Virtual Reality Workshop on K-12 Embodied Learning through Virtual \& Augmented Reality (KELVAR). 14, 2016, doi:10.1109/KELVAR.2016.7563674.

[12] E. Gibney, "DeepMind algorithm beats people at classic video games," Nature, 518, 465-466, 2015, doi:10.1038/518465a.

[13] F. Ymran, O. Akeem, S. Yi, "Gamification Design in a History E-Learning Context," in 2017 International Conference on Information, Communication and Engineering (ICICE). 270-273, 2017, doi:10.1109/ICICE.2017.8479194.

[14] D. E. Asher, A. Zaldivar, J. L. Krichmar, "Effect of Neuromodulation on Performance in Game Playing: A Modeling Study," in 2010 IEEE 9th International Conference on Development and Learning, 155-160, 2010, doi:10.1109/DEVLRN.2010.5578851.

[15] E. Klopfer, Augmented learning: research and design of mobile educational games, Cambridge, 2008.

[16] S. Deterding, "Gamification: designing for motivation," Interactions, 19(4), 14-17, 2012, doi:10.1145/2212877.2212883.

[17] E. Sanchez, V. Emin-Martinez, "Towards a Model of Play: an Empirical Study," in C. Busch (Ed.), Proceedings of the 8th European Conference on Games Based Learning, 2, 503-512, 2014.

[18] K. M. Kapp, The gamification of learning and instruction: Game-based methods and strategies for training and education, Pfeiffer \& Company, 2016.

[19] L. Sheldon, The multiplayer classroom: designing coursework as a game, Cengage Learning, 2011.
[20] J. Gee, The ecology of games: connecting youth, games, and learning, MIT Press, 2008.

[21] K. Hogan, M. Pressley, Scaffolding student learning: instructional approaches and issues, Brookline Books, 1997.

[22] W. Konen, "General Board Game Playing for Education and Research in Generic AI Game Learning," in 2019 IEEE Conference on Games (CoG), 18, 2019, doi:10.1109/CIG.2019.8848070.

[23] MIT Media Lab Scratch (2019, August 1), Scratch, Available at: scratch.mit.edu

[24] M. Mendonça, H. S. Kondo, L. B. de Souza, R. H. C. Palácios, J. P. L. S. de Almeida, "Semi-Unknown Environments Exploration Inspired by Swarm Robotics using Fuzzy Cognitive Maps," in 2019 IEEE International Conference on Fuzzy Systems (FUZZ-IEEE), 1-8, 2019, doi:10.1109/FUZZ-IEEE.2019.8858847.

[25] E. I. Papageorgiou, Fuzzy cognitive maps for applied sciences and engineering, Springer, 2014.

[26] R Brooks, "A robust layered control system for a mobile robot," IEEE Journal of Robotics and Automation, 2(1), 14-23, 1986, doi:10.1109/JRA.1986.1087032.

[27] A. M. El-Sherbini, M. A. Aboul-Dahab, M. M. Fouad and M. F. Abdelkader, "Distance learning during Covid-19: Lessons learned and Case studies from Egypt," in 2021 IEEE Global Engineering Education Conference (EDUCON), 1743-1748, 2021, doi:10.1109/EDUCON46332.2021.9454051.

[28] A. Wilby, E. Lo, "Low-Cost, Open-Source Hovering Autonomous Underwater Vehicle (HAUV) for Marine Robotics Research based on the BlueROV2," in 2020 IEEE/OES Autonomous Underwater Vehicles Symposium (AUV), 1-5, 2020, doi:10.1109/AUV50043.2020.9267913.

[29] C. Persello, L. Bruzzone, "Active and Semisupervised Learning for the Classification of Remote Sensing Images," IEEE Transactions on Geoscience and Remote Sensing, 52(11), 6937-6956, 2014, doi:10.1109/TGRS.2014.2305805. 